\title{
Prevalence of Hypocalcaemia in Term Neonates and the Clinical Characteristics of the Affected Neonates Admitted to the Neonatal Intensive Care Unit at the University Teaching Hospital in Lusaka, Zambia
}

\author{
Zyambo, KDZ1, ${ }^{2}$ Sinyangwe, $\mathbf{S 1},{ }^{2}$ Chabala, $C^{1,2}$ \\ 1. University Teaching Hospital, Department of Paediatrics and Child Health, P/B RW1X, Lusaka, Zambia \\ 2. The University of Zambia, School of Medicine Ridgeway Campus, Lusaka, Zambia
}

\begin{abstract}
Address of Correspondence:
Khozya D. Zyambo, University Teaching Hospital, Department of Paediatrics and Child Health, P/B RW1X, Lusaka, Zambia. Cell phone: +260-963-332800, E-mail: khozyazyambo@gmail.com
\end{abstract}

\begin{abstract}
Background: Anaemia is a public health problem globally affecting 293.1 million children and $28.5 \%$ of these children are in the sub Saharan Africa. The aim of this study was to determine the prevalence of anaemia and the associated risk factors of anaemia in children aged 6 months to 15 years admitted to the University Teaching Hospital. There have been no studies done at University Teaching Hospital to establish the burden of anaemia in children.
\end{abstract}

Methods: A cross sectional study was conducted from July 2016 to December 2016. 351 children were recruited through convenient sampling methods. Data collection sheet was used to collect socio demographic and anthropometry data. The prevalence, associated risk factors of anaemia, and morphological types of anaemia were determined after blood investigations were done. Data analysis was done using SPSS version 21.0. The association between predictors and outcome variables were measured by using by logistic regression and bivariate analysis. Ethical permission was obtained, consent from parents/guardians was taken and confidentiality was maintained.

Results: A total of 351 children were studied. The mean age was 3 years (IOR 2-7 years). $45.9 \%$ were females and $54.1 \%$ were males $(\mathrm{P}=0.12)$. The mean cell volume was 74. 5fL.Malnourised children were $37.9 \%$ among those who were anaemic as compared to $33.7 \%$ in the non-anaemic group. $7.4 \%$ children had positive malaria by rapid diagnostic test (RDT). $23.8 \%$ had a positive sickling test. The prevalence of anaemia was 161/351 (45.9\%). Mild, moderate, severe anaemia was 47/161(29\%), $86 / 161(53 \%)$ and $28 / 161(17 \%)$ respectively.
The age group 6 months to 5 years was the most affected with $59 \%$ mildly, $69.8 \%$ moderately and $71.4 \%$ severely anaemic. On bivariate analysis malnutrition, HIV, malaria, age and sex were not associated with anaemia and there was no statistical difference. Logistic regression analysis revealed that presence of haemoglobin $\mathrm{S}$ was the only risk factor independently strongly associated with Anaemia (CI-0.2-0.7), p value-0.001.

Conclusion: Anaemia is a health problem at University Teaching hospital and the under-five age group is the most affected. Predictors of mild, moderate and severe anaemia is sickle cell disease. Therefore, improving on early screening of sickle cell disease and investigating the role of iron deficiency anaemia are some of the strategies to be advocated.

Keywords: Anaemia, Prevalence, Risk factors, Zambia, Africa

\section{INTRODUCTION}

Hypocalcaemia in the first three days is known as Early Neonatal Hypocalcaemia (ENH). In contrast, hypocalcaemia occurring in the second week of life is referred to as Late Neonatal Hypocalcaemia (LNH) commonly caused by ingestion of cow's milk which has a high content of phosphates 1 .

In utero, active calcium transfer from the maternal circulation to the fetus is facilitated by parathyroid hormone (PTH) via a trans-placental calcium pump mainly during the third trimester of pregnancy. As a result, total serum calcium concentration in the fetus is higher than that of the mother with total serum calcium levels in umbilical cord blood reaching 2.5-2.75 Mmol/L2. However, 
Placental transfer stops abruptly after birth. Within the first few hours of life, the newborns undergo a physiological drop in serum calcium levels by 24-72 hours of age. Then the calcium level increases to normal values by the tenth day of life 3,4 . This homeostatic state is achieved by the tight regulation of the following factors; PTH secretion, dietary calcium intake, renal calcium reabsorption, skeletal calcium stores, and vitamin D levels4.

In high-risk neonates; low birth weight, birth asphyxia, and infants of diabetic mothers 5,6,7,8, early neonatal hypocalcaemia is usually worsened, thus necessitating treatment with calcium supplementation for at least the first 72 hours 8 .

Normal levels of serum calcium vary with the gestation age of the newborn. Hypocalcaemia is defined as ionized calcium $<1.22 \mathrm{Mmol} / \mathrm{L}$ or total serum calcium $<2.21 \mathrm{Mmol} / \mathrm{L} 8$ in term neonates. Preterm infants are generally not considered hypocalcaemia until serum total calcium values fall below $1.8 \mathrm{Mmol} / \mathrm{L} 9$.

Ionized calcium is essential for a number of biochemical processes in the body. These include blood coagulation as a co-factor, neuromuscular excitability, cell membrane integrity and function, and cellular enzymatic and secretory activity 1,2 . Therefore, hypocalcaemia in neonates is potentially a life-threatening condition and may present with signs of neuromuscular irritability; myoclonic jerks, twitching of muscles and extremities, seizures, and other non-specific signs like apnoea, cyanosis, high pitched cry and/or cardiac rhythm disturbances (prolonged QTc)1.

Prevalence of neonatal hypocalcemia has been observed to vary with gestational age, perinatal factors, neonatal and maternal comorbidities. In one European study, it was found to be as high as $39 \% 10$.

The University Teaching Hospital (UTH) Neonatal Intensive Care Unit, (NICU), Zambia's largest referral hospital based in Lusaka - the capital city, admits close to 4000 neonates per year with various neonatal conditions 11 . One of the important neonatal conditions observed in NICU is neonatal hypocalcaemia 11. Anecdotal reports have shown that severely ill neonates (asphyxia, meningitis, acute respiratory distress syndrome) presenting with seizures and hypocalcaemia concurrently, respond poorly to anti-epileptic drug therapy until several doses of calcium gluconate are given empirically.

The measurement of serum calcium levels in neonates admitted to NICU as guided by the NICU protocol is not routinely done at UTH-NICU. This is because the UTH laboratory infrequently stocks reagents to run the test. WHO acknowledges that serum ionized calcium measurement in hospital settings is less readily available than measurement of blood sugar12. This inability to run routine serum calcium levels has led to delays in diagnosing hypocalcaemia in affected neonates and subsequently its prompt management. Due to lack of evidence (University of ZambiaUNZA library and internet search - PubMed and Cochrane library, showed no records of studies done at UTH or around the region on this subject) of the burden of neonatal hypocalcaemia to do a cost-benefit analysis, the laboratory services may not have been compelled enough to prioritize the provision of this particular service.

Secondly, in-light of the lack of reagents, investigations for hypocalcaemia many times are prompted by clinical signs and/or non-response of convulsions to anticonvulsants. Babies with hypocalcaemia are therefore identified late and appropriate treatment is often delayed, as a result newborn babies are subjected to prolonged stay in hospital with increased risk of developing irreversible brain injury due to prolonged convulsions and also for acquiring nosocomial infections.

Hence, this study endeavoured to establish the extent of the problem of hypocalcaemia in term neonates and document the clinic features of affected neonates. The findings will then provide the evidence required to review and design NICU treatment protocols that will guide in the management of electrolyte imbalances. Further, the findings will provide the required evidence to support/justify routine (currently not being practiced) measurement of serum calcium in all high risk neonates admitted to NICU despite the associated high costs of the test thereby ensuring early identification of hypocalcaemia in at-risk neonates. This will facilitate early initiation of appropriate interventional measures to correct any electrolyte disorders in order to improve morbidity outcomes, reduce the duration of stay in hospital and consequently, in the long term, reduce the risk of neurological sequelae that may result from prolonged uncontrolled fits.

\section{MATERIALS AND METHODS}

This was a cross sectional study conducted at UTH NICU in Lusaka, Zambia. The hospital is known 
to admit an average of 4000 newborns per year, 2900 of which are term neonates 11 . The unit receives patients from the UTH labour ward and various health centres in Lusaka.

The study included all term (37 weeks or more by gestation age) neonates less than 5 days old admitted to the NICU for various neonatal conditions. These included referrals from the UTH labour ward and from health centres in Lusaka urban. The study excluded preterm neonates, small for gestation age and neonates with congenital abnormalities.

Simple random sampling methods were employed to recruit study participants. The study recruited every other neonate who met the eligibility criteria that would be admitted to NICU between 8 hours and 18 hours every day of the week over a period of 6 months. A total of 240 patients were screened over a period of six months (December 2014 to May 2015). Of these, 200 patients met the eligibility criteria but only 179 patients where successfully recruited into the study while the rest of the 21 were left out for various reasons such as declined consent and missing results. The study was enrolling 1 to 4 patients per day, between 08 hours to 18 hours from Monday to Sunday. There were a total of 179 term neonates enrolled to this study and only 174 participants with serum calcium results were finally analyzed. The sample size was calculated using Epi info version 3.5.1 at power of $80 \%$ with an assumed study population of 2900 term neonates admitted to NICU every year and the assumed neonatal hypocalcaemia prevalence of $40 \% 10$ with the worst expected sample population prevalence of $32.5 \%$. The sample size calculated was 164 using the automated formula on Epi info software. However, the actual number of research participants included in this study came to 174 neonates.

Ethical clearance was obtained from ERES CONVERGE IRB reference number 2014-June016 . The permission to conduct the study at UTH was granted by Department of Paediatrics and Child Health and the UTH management. The risks and benefits of the study were fully and clearly explained to the research participants as described in the consent form. The study was not without risks and these where highlighted to the research participants. They included, delayed hemostasis from the venipuncture site especially in neonates at risk of bleeding tendencies such as severe sepsis as well as repeated venipuncturesand anaemia in neonates. The anticipated benefits included free serum calcium tests, early detection of electrolyte abnormality and quick referral for appropriate care.

An information sheet was given and/or read to potential study participants before consenting/ declining to be in the study. The consenting process was undertaken in the preferred language of the potential participant. An impartial witness was present throughout the consenting process in cases were the potential participant was unable to read. Once the consent was obtained, the study questionnaire on both the maternal characteristics and the neonatal characteristics was administered. Clinical assessment on the neonate was done by conducting a physical examination to determine the clinical features such as convulsions, hypertonia or hypotonia that the neonates may have presented with. The clinical findings were then documented into the case forms. Before collecting blood samples for analysis, the mothers of the neonates who met the criteria were approached to give consent after which blood was obtained from the patients using needle and syringe. A maximum of $2 \mathrm{mls}$ of blood sample was collected in a lithium heparin container from the peripheral veins. The samples where then coded, packed in a courier cooler box and sent to the UTH laboratory for chemistry and haematology analysis using the chemistry analyzer and PENTIUM haematology analyzer respectively. The following tests were requested; corrected serum calcium, sodium, phosphate and magnesium. Other tests which were performed in the study included; haematocrit (using the haematocrit machine) and random blood sugar measured using the glucometer. The results once obtained were availed to the attending clinician for appropriate action and documented on to the case forms for each research participant.

A standardized data entry questionnaire was used for each research participant for collection of data and the data was entered and aggregated into an Epi Info database.Data were analyzed using the statistical software package SPSS version 21. All statistical tests were at 5\% significance level. The Independent Samples T-test was used to compare mean values between groups, the Pearson's chisquared and Fisher's exact test was used for comparison of proportions between groups. The relationship between study variables and outcome variable of interest (hypocalcaemia) was examined using logistic regression. Selection for logistic regression model was considered at level $\mathrm{P}<0.20$ or known clinical significance. 


\section{RESULTS}

\section{Characteristics of the Study Population}

The mean age of the study population was 1.3 days $(\mathrm{SD}=0.60)$ while the male to female ratio was $1.4: 1 ; 101 / 174(58 \%)$ male and 73/174 (42\%) female neonates. Sixty nine (39.7\%) neonates had birth weights ranging between 2.5 to $3.0 \mathrm{Kg}$ while $93(53.4 \%)$ were between 3.1 to $3.9 \mathrm{Kg}$ and 12 $(6.9 \%)$ were macrosomic, with birthweight over $4.0 \mathrm{Kg}$. The mean gestation age was $38.8 \pm 1.24$ weeks by dates.

The most common reason for referral to UTH NICU for the neonates was asphyxia 62/174 (35.6\%) followed by fever 25/174 (14.4\%) while the most common clinical diagnosis made in the admitted neonates was asphyxia 59 (33.9\%) and sepsis $58(33.3 \%)$. Figure 1 shows a bar chart for neonatal diagnosis and Table 1 shows the reasons for referral frequency distribution.

\section{Maternal Characteristics}

Maternal age had a mean of 24.9 years $(\mathrm{SD}=6.60)$ as shown in Table 2. Maternal parity was right skewed with a median of $1(\min =1, \max =8)$. There were $24(13.8 \%)$ mothers with a positive HIV status and $150(86.2 \%)$ had a HIV negative status. There were only $5(2.9 \%)$ mothers with diabetes mellitus while a majority $169(97.1 \%)$ did not have diabetes mellitus.

\section{Laboratory Findings - Prevalence of hypocalcaemia}

Of the 179 neonates enrolled in to the study, 174 had corrected serum calcium test results available for analysis. Figure 2 shows 98 (56.3\%) neonates had normal calcium levels, $46(26.4 \%)$ had hypocalcaemia and the rest had hypercalcaemia. Table 3 shows summary statistics of the rest of laboratory tests done on the neonates in this study.

\section{Findings of the Clinical Features - Convulsions}

There were $89 / 174 \quad(51.1 \%)$ neonates with abnormal neurological clinical features (convulsions, hypertonia and hypotonia). Figure 3 shows the frequency distribution of the abnormal neurological features found in the neonates. The most common abnormal neurological feature seen in the study were convulsions, 71/174 (40.8\%), followed by hypertonia $14(8.0 \%)$.

Further analysis of the results revealed that of the 46 neonates with hypocalcaemia in this study 23
$(50 \%)$ of the neonates had abnormal neurological features. There was however no statistically significant association between hypocalcaemia and convulsions $(\mathrm{P}=0.99)$ as shown in table 6 .

\section{Bivariate Analysis for Categorical and Continuous Variables}

At 5\% significance level the clinical diagnosis of the neonate was associated with hypocalcaemia, $\mathrm{P}$ $<0.01$ and magnesium was marginally associated with hypocalcaemia, $\mathrm{P}=0.06$. It was observed that

$19 / 71(21.3 \%)$ of the neonates with convulsions also had hypocalcemia (Table 6). However, there was no statistically significant association between overall neurological status of the neonate and hypocalcaemia $(\mathrm{P}=0.86)$ and neither was there significant association between actual neurological disorder and hypocalcaemia $(\mathrm{P}=0.60)$.

\section{Multivariate Logistic Regression Analysis}

Child age, gestation age, sodium, magnesium, and clinical diagnosis were entered into a multivariate logistic regression model and the backward selection method executed. The resulting model predicting hypocalcaemia is shown in Table 7 .

Adjusting for gestation age and clinical diagnosis the odds for hypocalcaemia increased 3 times for every lunit increase in magnesium level $(\mathrm{OR}=3.11, \mathrm{CI}=1.24-7.82, \mathrm{P}=0.02)$. Adjusting for magnesium and clinical diagnosis the odds for hypocalcaemia reduced by $27 \%$ for every 1 weekincrease in gestation age $(\mathrm{OR}=0.73, \mathrm{CI}=$ $0.52-1.03, \mathrm{P}=0.07)$. Adjusting for magnesium levels and gestation age, neonates with a clinical diagnosis of electrolyte imbalance had 11 times increased odds for hypocalcaemia compared to neonates with other clinical diagnoses $(\mathrm{OR}=$ 11.33, CI $=2.69-47.77, \mathrm{P}<0.01)$. Although not statistically significant, neonates with asphyxia had $24 \%$ increased odds for hypocalcaemia (OR $=1.24, \mathrm{CI}=0.43-3.74, \mathrm{P}=0.73$ ) compared to neonates with other clinical diagnoses. Neonates with sepsis (clinical diagnosis) had 2 times increased odds for hypocalcaemia $(\mathrm{OR}=2.06$, $\mathrm{CI}=0.65-6.56, \mathrm{P}=0.22$ ) compared to neonates with other clinical diagnoses, however this was not statistically significant.

\section{DISCUSSION}

The findings seem to suggest a high prevalence of hypocalcaemia in the neonates admitted with abnormal neurological findings, which correlates 
well with what has been shown in literature10.A recent retrospective study by Cho 13 found that $36 \%$ of the neonates with hypocalcaemia had tetanic spasms and increased tremulousness as the most common clinical features while Roberton 10 found $17.6 \%$ of the neonates had jitteriness and irritability with a majority of the hypocalcaemic neonates not having any neurological findings. Even though this study managed to document neurological abnormalities in the hypocalcaemic neonates, we did not observe some of the characteristic neurological findings of neonatal hypocalcaemia as described in most literature 10,13. This could be attributed to the study design which did not provide for follow up reviews on the neonates other than a single encounter during recruitment. As a result, some of the clinical findings in the newborns could have been missed.

Fifty-one percent of the neonates in this study had abnormal neurological findings, 79.8\% (71/89) of whom had overt convulsions. Albeit no statistically significant association was seen between neurological disorder and hypocalcaemia. Multiple etiologies for convulsions often co-exist and a comprehensive evidence based approach for their management cannot bethis cannot be overemphasized hence the non-statistical significance as asphyxia could have caused the seizures including sepsis14. It is imperative to take note of the fact that hypocalcaemic neonates also tend to have convulsions that are resistant to treatment with anti-epileptic drugs, hence the dire need for early establishment of serum calcium levels in all neonates, especially the ones that are fitting, in order to promptly intervene with appropriate therapy. Prophylactic administration of calcium gluconate to all high risk neonates who present with convulsions has been found to be beneficial and may contribute to reduction in morbidity and duration of hospital stay of the neonates 10.

The high number of hypocalcaemic and convulsing neonates seen at UTH NICU may further be explained by the high numbers of very ill neonates that are admitted to the unit. We found that $35.6 \%$ of the neonates admitted were referred for asphyxia, $14.4 \%$ for fever, $10.3 \%$ for convulsions and $9.2 \%$ for suspected sepsis. These findings correlate with the final clinical diagnoses made in the neonates i.e. $33.9 \%$ had asphyxia, $33.3 \%$ sepsis and $9.8 \%$ with electrolyte imbalance. These stressful neonatal events are known to induce elevated levels of stress hormones (corticosteroids, calcitonin and glucagon) which in turn aggravate low parathyroid hormone levels leading to impaired serum calcium mobilization15,16. In addition, these severely ill neonates have poor or no oral intake of calcium from their mothers while the ongoing metabolic dysregulation and the resultant acidaemia further increases the risk of hypocalcaemia10. This is supported by the results obtained from further data analysis which showed that the overall clinical diagnoses were associated with hypocalcaemia.

We also observed that the diagnosis of asphyxia and neonatal sepsis made up two thirds of the neonatal conditions seen in the study. It was even noted that the neonates with asphyxia had 24\% increased risk for hypocalcaemia when compared to neonates with other clinical diagnoses. This is significant in that asphyxiated neonates tend to have deranged electrolyte balance system worsened by the state of renal hypo-perfusion. Neonates with sepsis had 2 times increased odds for hypocalcaemia compared to neonates with other clinical diagnoses.

Neonatal hypocalcaemia is known to be exacerbated by hypomagnesaemia, however, it was not a prominent feature in our study as there were only $8 \%$ of the neonates with hypomagnesaemia. There was marginal association between serum magnesium levels and hypocalcaemia although it was not statistically significant.

The mean maternal age was 24.9 years and the majority of the mothers were primi-parous. This observation unveils a finding of great significance from which important inferences can be made in view of the observed high occurrence of neonatal medical complications born to the first time young mothers. This raises pertinent questions that need answers through further research; are the neonates born from first time young mothers at increased risk of developing neonatal complications and consequently hypocalcaemia? Or perhaps is there an underlying micro-nutrient deficient status in first time young mothers that maybe having an effect on the serum calcium levels of their newborns? Literature has shown that during pregnancy, mothers provide large amounts of calcium to the developing fetus except in instances of maternal hypo/hyperparathyroidism or vitamin $\mathrm{D}$ deficiency disorders 17 . Vitamin D deficiency in infants and mothers is a re-emerging public health issue. In a Canadian study, they found almost half of healthy mothers $(46 \%)$ and one third $(36 \%)$ of their newborn term infants had plasma 25-hydroxy vitamin D levels consistent with deficiency 18 . Risk factors associated with neonatal hypocalcaemia 
resulting from vitamin D deficiency which is due to maternal hypovitaminosis $\mathrm{D}$ include a dark skinned mother, low dietary Vitamin D maternal intake during pregnancy and lactation, and lack of maternal sun exposure 18 .

\section{STUDY LIMITATIONS}

The inability to determine the actual causes of convulsions through exhaustive investigations among the neonates made it difficult to make associations. The causes of convulsions in neonates are multi-factorial. Other than hypocalcaemia, the study was unable to account for alternative causes hence difficult to completely associate convulsions with hypocalcaemia.

\section{CONCLUSION}

The prevalence of hypocalcemia in term neonates admitted to NICU at UTH is high with one in every four newborns being affected. Half of the hypocalcaemic neonates manifest neurological features and convulsions are the most frequent. Only a quarter of the neonates who presented with convulsions in this study were found to have concurrent hypocalcaemia.

\section{ACKNOWLEDGEMENT}

This research study was mainly funded by the government sponsored student project allowance and additional financial assistance was provided by UNZA-MEPI. The UTH provided the laboratory support at a reasonable fee.

\section{REFERENCES}

1. Wandrup J, Kroner J, Pryds O, Kastrup KW. Age-related reference values for ionized calcium in the first week of life in premature and full-term neonates. Scand Journal Clinical Laboratory Investigations, 1988; 48:255.

2. Wandrup J. Critical Analytical and Clinical Aspects of Ionized Calcium in Neonates. Clinical Chemistry, 1989; 35/10, 2027-2033.

3. Loughead JL, Mimouni F, Tsang RC. Serum ionized calcium concentrations in normal neonates. American Journal Diseases of Childhood, 1988; 142:516.

4. Husain SM, Veligati N, Sims DG, et al. measurement of ionized calcium concentration in neonates. Archives of Diseases of Childhood, 1993; 69:77.
5. Tsang R, Steichen J.J, Chan G.M. Neonatal Hypocalcaemia; Mechanism of Occurrence and Management. Critical care medicine, 1977; 5; 1; 56-61.

6. Tsang R.C, William O. Neonatal Hypocalcaemia in Low Birth Weight Infants. Paediatrics, 1970; 45; 773.

7. Tsang RC, Chen IW, Friedman MA, Chen I. Neonatal parathyroid function: role of gestational age and postnatal age. J Pediatr 1973;83:728-38.

8. Jain A, Agarwal R, Sankar M. J, Deorari A. K, Vinod K. P. Hypocalcaemia in the Newborn. Indian Journal of Paediatrics, 2008;75 (2): 165-169.

9. Zhou P, Markowitz M. Hypocalcemia in Infants and Children.Pediatrics in Review 2009; 30; 190.

10. Roberton N.R.C, Smith M.A. Early Neonatal Hypocalcaemia. Archives of disease in childhood.1975; 50: $604-609$.

11. Medical Records, Department of Paediatric and Child Health, University Teaching Hospital, 2013 - 2015.

12. WHO, 2011. Guidelines on neonatal seizures. ISBN 9789241548304.

13. Cho W, Yu HW, Chung HR, Yang SW, Choi CW, Kim BI. Clinical and Laboratory Characteristics of neonatal hypocalcaemia. Ann Pediatr Endocrinol Metab. Journal, 2015;20(2):86 - 91.

14. Sankar JM, Agarwal R, Deorari A, Paul VK. Management of Neonatal Seizures. Indian J Pediatr 2010;77:1129-35.

15. Cruikshank DP, Pitkin RM, Reynolds WA, Williams GA, Hargis GK. Effects of Magnesium Sulphate Treatment on Perinatal Calcium Metabolism. 1. Maternal and Fetal Responses. Am J Obstet Gynecol, 1979 Jun 1;134(3):243-9.

16. Bergman L, Kjellmer I, Selstam U. Calcitonin and Parathyroid Hormone - Relation to Early Neonatal Hypocalcaemia in Infants of Diabetic Mothers. Biol Neonate 1974;24:151-160.

17. Kovacs CS, Vitamin D in Pregnancy and Lactation: maternal, fetal, and neonatal outcomes from human and animal studies. The American Journal of Clinical Nutrition,2008;88 (suppl):520S-8S.

18. Shuman R, O'Gorman CS, Sochett EB. Case 1: Neonate with Seizures and Hypocalcaemia. Paediatric Child Health, 2008;13(3): 197 - 200. 


\section{TABLES AND FIGURES}

Table 1: Reasons for Referral Frequency Distribution

\begin{tabular}{|c|c|c|c|}
\hline Reason For Referral & Frequency & Percent $\%$ & \\
\hline Asphyxia & 62 & 35.6 & \\
\hline Fever & 25 & 14.4 & \\
\hline Convulsions & 18 & 10.3 & \\
\hline Sepsis & 16 & 9.2 & \\
\hline Fast breathing & 12 & 6.9 & \\
\hline Big baby & 9 & 5.2 & \\
\hline Hypothermia & 6 & 3.4 & \\
\hline Birth trauma & 5 & 2.9 & \\
\hline Aspiration & 4 & 2.3 & \\
\hline Infant of a diabetic mother & 3 & 1.7 & \\
\hline Post mature & 3 & 1.7 & \\
\hline Other & 11 & 6.3 & \\
\hline TOTAL & 174 & 100 & \\
\hline Test & $N$ & Standard Deviation & Median \\
\hline Random blood sugar & 174 & 3.40 & 4.70 \\
\hline Calcium & 174 & 0.39 & 2.18 \\
\hline Magnesium & 171 & 0.39 & 0.95 \\
\hline Phosphate & 148 & 1.23 & 2.31 \\
\hline Sodium & 163 & 9.49 & 139 \\
\hline Haematocrit & 174 & 6.39 & 49 \\
\hline Platelets & 144 & 93.09 & 245 \\
\hline Neutrophils & 103 & 5.49 & $11.30 \times 10^{3}$ \\
\hline Leucocytes & 144 & 7.25 & $5.10^{3}$ \\
\hline
\end{tabular}

Table 2. Summary Statistics of Laboratory Test Results

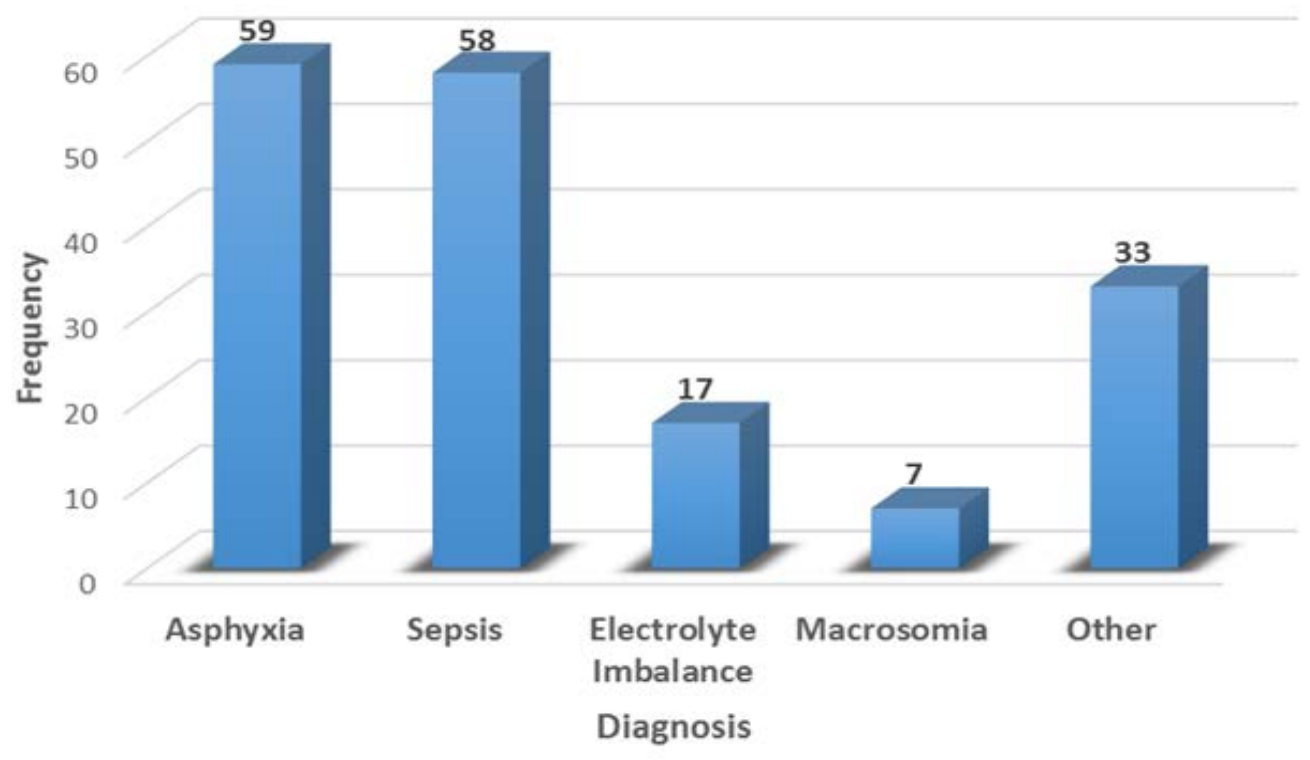

Figure 1: Neonatal Diagnosis Bar Chart 


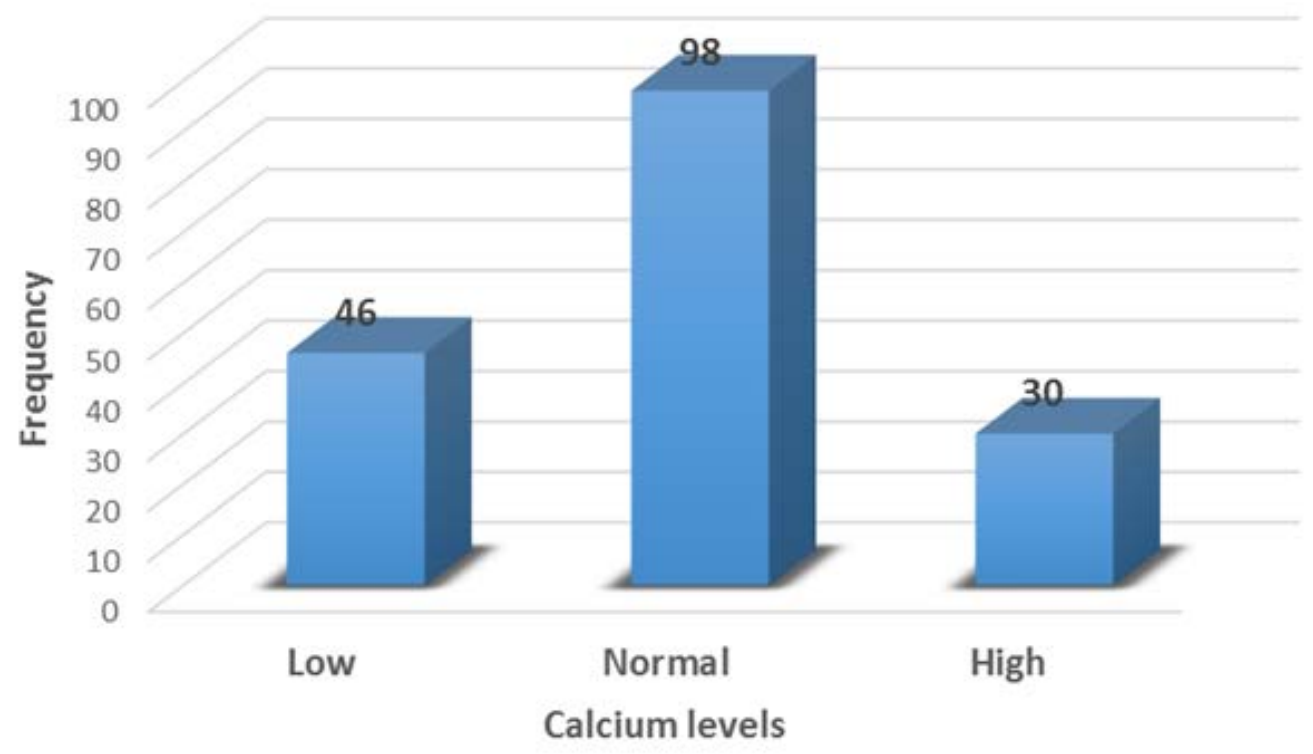

Figure 2: Frequency of Neonate Serum Calcium Levels Bar Chart

Table 3. Categorical Variables Bivariate Analysis

\begin{tabular}{|c|c|c|c|c|c|}
\hline \multirow[t]{2}{*}{ Variables } & \multicolumn{2}{|c|}{ Hypocalcaemia } & \multicolumn{2}{|c|}{ No Hypocalcaemia } & \multirow[t]{2}{*}{ P-Value } \\
\hline & $n$ & $\%$ & $n$ & $\%$ & \\
\hline \multicolumn{6}{|l|}{ Sex } \\
\hline Male & 26 & $56.5 \%$ & 75 & $58.6 \%$ & 0.81 \\
\hline Female & 20 & $43.5 \%$ & 53 & $41.4 \%$ & \\
\hline \multicolumn{6}{|l|}{ HIV status } \\
\hline Negative & 41 & $89.1 \%$ & 109 & $85.2 \%$ & 0.50 \\
\hline Positive & 5 & $10.9 \%$ & 19 & $14.8 \%$ & \\
\hline \multicolumn{6}{|l|}{ Maternal parity } \\
\hline One & 21 & $45.7 \%$ & 68 & $53.1 \%$ & 0.38 \\
\hline Two or more & 25 & $54.3 \%$ & 60 & $46.9 \%$ & \\
\hline \multicolumn{6}{|l|}{ Neurology } \\
\hline Normal & 23 & $50.0 \%$ & 62 & $48.4 \%$ & 0.86 \\
\hline Not normal & 23 & $50.0 \%$ & 66 & $51.6 \%$ & \\
\hline \multicolumn{6}{|l|}{ Diagnosis } \\
\hline Electrolyte imbalance & 11 & $23.9 \%$ & 6 & $4.7 \%$ & $<0.01$ \\
\hline Asphyxia & 13 & $28.3 \%$ & 46 & $35.9 \%$ & \\
\hline Sepsis & 16 & $34.8 \%$ & 42 & $32.8 \%$ & \\
\hline Macrosomia & 0 & $0.0 \%$ & 7 & $5.5 \%$ & \\
\hline Other & 6 & $13.0 \%$ & 27 & $21.1 \%$ & \\
\hline
\end{tabular}


Table 4. Continuous Variables Bivariate Analysis

\begin{tabular}{lccc}
\hline \multicolumn{1}{c}{ Variables } & $\begin{array}{c}\text { Hypocalcaemia }(\boldsymbol{n}=\mathbf{4 6}) \\
\text { mean }(\mathbf{S D})\end{array}$ & $\begin{array}{c}\text { No hypocalcaemia }(\boldsymbol{n}=\mathbf{1 2 8}) \\
\text { mean }(\boldsymbol{S D})\end{array}$ & P-alue \\
\hline Age & $1.4(0.75)$ & $1.3(0.54)$ & 0.22 \\
\hline Birth weight & $1.7(0.60)$ & $1.7(0.61)$ & 0.97 \\
\hline Gestation age & $38.54(1.07)$ & $38.8(1.30)$ & 0.19 \\
\hline Haematocrit & $48.6(7.69)$ & $48.7(5.89)$ & 0.88 \\
\hline Platelets & $236.2(125.50)$ & $249.2(80.47)$ & 0.57 \\
\hline Sodium & $135.8(13.52)$ & $139.0(7.38)$ & 0.15 \\
\hline Phosphate & $2.5(1.06)$ & $2.7(1.30)$ & 0.31 \\
\hline Magnesium & $1.19(0.51)$ & $1.03(0.34)$ & 0.06 \\
\hline Random blood sugar & $5.8(3.54)$ & $5.8(3.37)$ & 0.95 \\
\hline
\end{tabular}

Table 5. Frequency Distribution of Abnormal Neurological Features

\begin{tabular}{|c|c|c|c|c|c|}
\hline \multirow{2}{*}{$\begin{array}{l}\text { Neurology disorder } \\
\qquad(\mathrm{n}=\mathbf{8 9})\end{array}$} & \multicolumn{3}{|c|}{ Hypocalcaemia } & \multirow[t]{2}{*}{ Total } & \multirow[t]{2}{*}{ P value } \\
\hline & & Yes & No & & \\
\hline \multirow[t]{2}{*}{ Convulsions } & $\mathrm{N}$ & 19 & 52 & 71 & 0.99 \\
\hline & $\%$ & $26.8 \%$ & $73.2 \%$ & $100.0 \%$ & \\
\hline \multirow[t]{2}{*}{ Hypotonia } & $\mathrm{N}$ & 1 & 3 & 4 & - \\
\hline & $\%$ & $25.0 \%$ & $75.0 \%$ & $100.0 \%$ & \\
\hline \multirow[t]{2}{*}{ Hypertonia } & $\mathrm{N}$ & 3 & 11 & 14 & \\
\hline & $\%$ & $21.4 \%$ & $78.6 \%$ & $100.0 \%$ & \\
\hline \multirow[t]{2}{*}{ Total } & $\mathrm{N}$ & 23 & 66 & 89 & \\
\hline & $\%$ & $25.8 \%$ & $74.2 \%$ & $100.0 \%$ & \\
\hline
\end{tabular}

Table 7: Multivariate Logistic Regression Predicting Hypocalcaemia

\begin{tabular}{lccc}
\hline \multicolumn{1}{c}{ Variable } & Crude odds ratio (95\% ci) & Adjusted odds ratio (95\% ci) & P-value \\
\hline Magnesium & $2.56(1.12-5.85)$ & $3.11(1.24-7.82)$ & 0.02 \\
\hline Gestation age & $0.83(0.62-1.10)$ & $0.73(0.52-1.03)$ & 0.07 \\
\hline Diagnosis & 1 & & \\
\hline Other & & 1 & $<0.01$ \\
\hline Electrolyte imbalance & $8.25(2.18-31.23)$ & $11.33(2.69-47.77)$ & 0.73 \\
\hline Asphyxia & $1.27(0.43-3.74)$ & $1.24(0.37-4.11)$ & 0.22 \\
\hline Sepsis & $1.71(0.60-4.93)$ & $2.06(0.65-6.56)$ & \\
\hline
\end{tabular}

